\title{
Designing Augmented Reality Applications for Personal Health Decision-Making
}

\author{
Francisco Gutiérrez \\ KU Leuven \\ francisco.gutierrez@cs.kuleuven.be \\ Robin De Croon \\ KU Leuven \\ robin.decroon@kuleuven.be
}

\author{
Sven Charleer \\ KU Leuven \\ sven.charleer@kuleuven.be
}

KU Leuven

nyinyi.htun@cs.kuleuven.be

\author{
Katrien Verbert \\ KU Leuven \\ katrien.verbert@cs.kuleuven.be
}

\begin{abstract}
Augmented reality $(A R)$ is a technology that can assist with our daily decision-making tasks by presenting information that extends the physical world. However, little work has been done to understand the effect of the layout of AR interfaces on decision-making. In this paper, we present PHARA, an AR-based personal assistant that supports decision-making for healthier food products. In a controlled user study $(n=28)$, we explored the use of four different AR layouts on two different devices: Microsoft HoloLens and smartphone. Using subjective and objective means, we measured their effects on decision-making tasks that occur when people hold food products in their hands. We found that pie and grid layouts perform better on the smartphone, whereas a stacked layout works better on the reduced field-of-view of the Microsoft HoloLens, potentially at the cost of some affordances such as time spent and actions.
\end{abstract}

\section{Introduction}

Augmented reality (AR) creates an immerse experience by superimposing virtual objects upon physical objects in the real world and generates an illusion for the observer that such virtual objects exist in the physical space [1]. There are many devices available to deploy AR applications [2]: the most popular ones are head-mounted displays (HMD) and handheld experiences. HMD devices provide a full immerse experience, where virtual objects surround the users and augment their perception. Handheld experiences, on the other hand, typically work like a magic lens [3], where users use their smartphone screen and camera to discover virtual objects in the physical world. In 2016, Pokémon Go demonstrated the success of an AR handheld experience [4], but also showed that fulfilling complete immersiveness will likely take many more years to achieve. Applications for AR go beyond entertainment and include domains such as architecture, tourism, and medicine. In our work, we are interested in AR applications in the field of personal health and food which has been identified as an important future trend of AR technology [5]. AR virtual components can provide much more useful and comprehensive information related to food products, as well as the user's health profile and food interests. Food labels are considered an essential element in strategies against unhealthy diets and obesity [6]. Thus, AR can be useful at a critical moment of decision, when users hold the products in their hands. The objective of our work is to assist users by providing rich visual components that support their decision-making process.

So far, research has focused on how to display AR virtual components around physical objects and their interactions [7]. To the best of our knowledge, little work has been done to understand the impact of the layout of AR interfaces. Previous user studies show that users will change their behavior depending on how a system presents information. For instance, Madsen et al. [8] tested different AR labeling layouts to display annotations around physical objects. They found that users can more easily find objects with labels that stay static in the 3D space relative to the physical object. They indicate that AR systems should avoid updating labels positioning after their initial placement from the current viewpoint. Moreover, Chen and Tsoi [9] tested different layouts for recommender system interfaces and their influences under user's decision process, using objective and subjective measures. They compared three typical layouts used in recommender systems: a list, a grid, and a pie layout. Results indicate that users reached more areas of content and had a greater confidence and enjoyability while using the pie layout. In this paper, we research specifically how different layouts for representing recommendations affect decision-making 


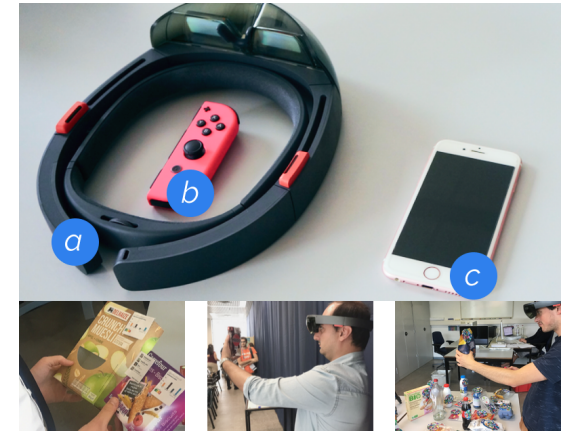

Figure 1. Top: PHARA: a) HoloLens, b) HoloLens input. c) iPhone 6S; Bottom: Design process.

in AR interfaces. To that end, we pose the following research question:

RQ: Regarding subjective and objective factors: what are the benefits and tradeoffs of using popular layouts such as, stack, grid, list and pie in AR applications within handheld and HMD experiences to support decision-making?

To address this question, we implemented the Personal Health Augmented Reality Assistant (PHARA), a system that supports decision-making about food products. It extends the information labelled on food products. To be specific, when a user holds a product in their hand, PHARA displays different AR-based virtual cards around the physical food product, presenting alternative products and other useful information related to the product considering the user's health. Furthermore, we conducted a controlled lab study focused on food selection tasks. Measuring subjective and objective means, we asked 28 participants to complete a set of tasks using four layouts: stack, list, grid and pie (see Figure 3), and two implementations of PHARA: PHARA HMD that uses the HoloLens and PHARA handheld that uses an iPhone 6S, see Figure 1 (top). Our contributions are as follows: 1) we propose a set of AR layouts to display virtual content around physical objects in the real world, particularly focusing on a number of popular layouts such as a pie, grid, list and stack; 2) we identify, through a user study, which of the four layouts are the most affordable with HMD and handheld AR experiences in a decision-making scenario.

\section{Related Work}

\subsection{Recommender systems for health}

In recent years, the application of recommendation techniques in health has attracted increased interest [10]. Objectives include delivering relevant information to end-users that is trustworthy [11], lifestyle change recommendations [12], and improving patient safety [13]. The latter category, for instance, includes research on how to use recommender systems to suggest relevant information about interactions between different drugs, in order to avoid health risks.

Lifestyle change recommendations focus among others on suggesting users how to improve their eating, exercising or sleeping behavior [14, 15]. An example is to use recommendations as a basis to algorithmically derive balanced meal plans that meet nutritional guidelines for the user [14]: these recommendations are based on a personal profile, such as gender, height, weight, physical activity, and estimate an individual's basal metabolic rate and daily kilocalorie requirements. Similar objectives have been researched by van Pinxteren et al. [16] and Achananuparp and Weber [17], who explored healthy food recommendations by finding food substitutes using a crowdsourced service. We focus specifically on the use of recommendation techniques to improve eating behavior. We build on the work of [14] to recommend healthy food products to casual end-users. As in work of Achananuparp and Weber [17], we use a crowd-sourced database to retrieve product information. As the selection of food products is a decision users often make in grocery stores, we research the application of recommender techniques with both a smartphone and the HoloLens in an AR experience.

\subsection{Recommendations for food product selection}

The idea to support users during the selection of food products is not new. iGrocer [18] is a grocery shopping assistant that has been proposed to assist users, and in particular older users and shoppers with disabilities, in deciding which products to buy and which products to avoid based on nutrition criteria. The system relies on a content-based recommendation approach and has been implemented for use on smartphones. The system also calculates the shortest path in the grocery store to find the products. To the best of our knowledge, the proposed system has not been evaluated with end-users.

Ahn et al. [19] extend this concept and developed a handheld AR system that provides the user with recommendations based on nutrition criteria and calorie intake. The system is also implemented for use on smartphones and annotates products with tags that indicate which products to buy, and which products to avoid. Evaluation results with 15 participants indicate that the application reduced the amount of time for finding healthy food products and that the tag coloring 
helped users to identify and avoid unhealthy products.

In this paper, we further expand upon these concepts, and present the evaluation results of an AR system for both smartphones and HMD devices that recommends products to end-users when they hold the products in their hands. We use different visualizations to represent recommendations of healthy products, and to enable users to inspect nutritional facts and the number of calories compared to their recommended daily calorie-intake. The approach has been identified as an important strategy that motivates users to eat healthier [20]. This strategy keeps the user involved in the decision-making process, while at the same time improves the user's ability to choose healthy products. We evaluate different layouts that visualize recommendations and nutritional facts to support decision-making. We also compare which of these layouts work better for use on handheld and HMD devices.

\subsection{Layout Design}

Research about layout design for AR systems exists in different application areas. ARLive [21] is an application that enables users to interact with physical objects in the real world. By using an internet-of-things-based approach, the system displays different visual components around the physical object. The user can interact with the virtual interfaces to change the state of the physical object in real-time. ARLive provides no particular layout to the interface. Objects appear to be organized only around the target. [8] tested different AR labelling layouts to display information around physical objects. They found that 3D space is essential and labels should be treated as $3 \mathrm{D}$ objects that take part of the scene. Integrating 3D labels into the scene allows the AR system to apply the camera pose to the labels naturally, and also simplifies the design of 3D interaction methods. The authors found that users perform better with labels that stay static in the $3 \mathrm{D}$ space relative to the physical object. AR systems should avoid updating labels positioning after their initial placement from the current viewpoint.

In this paper, we explore the use of different layouts to represent recommendation results in $\mathrm{AR}$ experiences. We build upon the work of [9] for serving recommendations to end-users. Chen and Tsoi [9] tested the influences of different layouts on desktops, including list, grid, and pie layouts. The results indicate that users reached more areas of content and had greater confidence and enjoyability when using the pie layout. We evaluate whether these layouts can be used in AR experiences to support decision-making of end-users.
The research contribution is two-fold: first, we transfer the idea of different layouts to AR implementations that can be used to support in-situ recommendations. Second, we evaluate whether HMD devices, such as the HoloLens, can also be used to support decision-making for non-expert users, and compare the utility and effectiveness of the different layouts on both smartphone and HoloLens devices for decision-making about food products.

\section{System Design}

The design of PHARA was inspired by related work and a preliminary study that is summarized in the next subsection. We used this input to refine our design process.

Preliminary studies: during the design process of PHARA, we conducted several user studies. At an early stage, we evaluated a paper prototype [22], followed by a working prototype at a conference venue. The different stages of development are represented in Figure 1,bottom. During the paper prototype evaluation, we printed and tested different visual components that we wanted to integrate into our system to augment food products. With the early prototype, we learned that more detailed information was required, such as calorie breakdown, and that the introduction of personal information would be useful to make users aware of the impact of a food product in their health. With this initial feedback, an early working prototype was developed with the HoloLens. We tested our application at a conference venue $(n=10)$ where we received feedback from interested people that used the system. We discovered that, due to hardware restrictions, some design variables would be necessary to reconsider in the system, such as scaling the visuals and the layout to the device limited display area. The initial prototype used voice commands and gestures as input method, but users reported to feel uncomfortable when interacting with the system, particularly in a public space. Many participants suggested also the use of a handheld device to interact with our system. All of these comments gathered during our preliminary studies were useful towards defining the final design of PHARA.

Design requirements: we imagine the following scenario: a user goes to the grocery store to buy some food products for dinner. The user can use PHARA on his/her smartphone or the store can provide an HMD device, which can be used during the grocery store visit. The system enables users to get additional information about the products they hold in their hands, including nutritional facts, and enables them to inspect the number of calories compared to personal daily-intake. At the 
end of the experience, users can reflect on the products they selected and use the system to see if they would have a balanced meal. They can also replace products with similar or healthier alternatives. This last aspect of the scenario makes it particularly important to consider different layouts for the design of PHARA. For example, while a stacked layout may be useful in AR because of the use of the three-dimensional space to organize information in layers, a pie or grid layout might be more useful to see all the information distributed around the food product. As mentioned earlier, a key objective is to keep the user involved in the decision-making process by visualizing relevant information that shows the impact on the user's health. To summarize, our design requirements are the following: (1) Show a visual card system that shows information of a product when users hold this product in hand. (2) Provide recommendations based on similar products, healthy alternatives and food products the user may like. (3) Show different layouts to explore the visual cards from the system. (4) Show information about the impact of the food product using data about the user.

\section{Implementation}

Following the design requirements, we decided to implement PHARA for use on both handheld and HMD devices. We created an AR application using Unity and Vuforia Framework ${ }^{1}$, allowing us to deploy an application for the HoloLens and the iPhone. We also used the Vuforia AR library to register food products and created markers to track and recognize the food products. Following a client-server architecture, we implemented a system to serve both PHARA handheld and PHARA HMD experiences.

Server-side: we used a dump from the Open Food Facts $^{2}$ database, filtered for products that match our region. Open Food Facts is a crowd-sourced database with more than 10,000 food products from around the world. It contains information about packaging, brand, category, list of ingredients, food additives and nutritional information, making it very relevant for the purposes of PHARA. Moreover, we created a database in MongoDB to store personal information of the users in order to support them with information related to their profile. To provide recommendations to the users, we implemented a content-based recommender engine using a Python API. Products from the food products database were compared between pairs using a cosine similarity metric, which is commonly used for providing product recommendations [23]. A similarity index was

\footnotetext{
${ }^{1}$ https://unity3d.com/partners/vuforia

${ }^{2}$ https://world.openfoodfacts.org
}

generated for each of the products in the database, keeping the top-four similar products to provide a ranked list with recommendations for the user. The server also included a prediction component, which uses the data from the user's profile to estimate their calorie intake. Using a Python script, we estimated the metabolic rate (BMR) and daily kilo-calorie requirements of the user based on the Harris-Benedict equations revised by Mifflin and St Jeor [24]. Based on this information, the prediction component also estimated the number of calories required to keep their current weight and estimation of weight gain/loss in the following three months.

Client-side: we created a basic registration Web application. In this application, users can create a profile by entering their basic information such as age, weight, gender, and activity level. Users can also select products of their interest. Following our design guidelines and feedback from previous studies, we implemented various visual components together with the different layouts. Inspired by the material design guidelines ${ }^{3}$, we designed all the required visual interfaces as card components, as presented in Figure 2. Cards are visual components to display concrete and organized information to the user. The following cards were implemented in PHARA:

Figure 2a, Nutrition Levels: a bar chart shows an overview of the concentrations of fats, saturated fats, salt and sugars based on a $100 \mathrm{~g} / \mathrm{ml}$ portion. The nutritional score (nutri-score) is a five-color nutrition label based on a color-coded scale that indicates the overall nutritional quality of the product [25]. We used the food products database to get the nutri-score for each of the products ${ }^{4}$.

Figure 2b, Calorie Breakdown: using the profile information of the user and calorie data of the product from Open Food Facts, a calorie breakdown is shown. The calorie breakdown card uses a 100-squares plot to represent the total amount of the daily calorie consumption of a user. A stacked bar with three-color scale represents the amount of carbohydrates, fats, and proteins included in the number of calories.

Figure 2c, Calorie Intake: we show an estimation of the variance of the weight of a user in the following three months, with encoded uncertainty, if a user increases or decreases the amount of calorie consumption by 500 kcal.

Figure $2 d$, Nutrition Guide: inspired by the "Healthy Eating Plate" created by experts from Harvard School of the Public Health and Harvard Medical School [26], we created a bar chart with guidelines about portions of vegetables, proteins, fruits, grains, and water for a

\footnotetext{
${ }^{3}$ https://material.io/guidelines/components/cards.htm

${ }^{4}$ https://fr.openfoodfacts.org/score-nutritionnel-france
} 

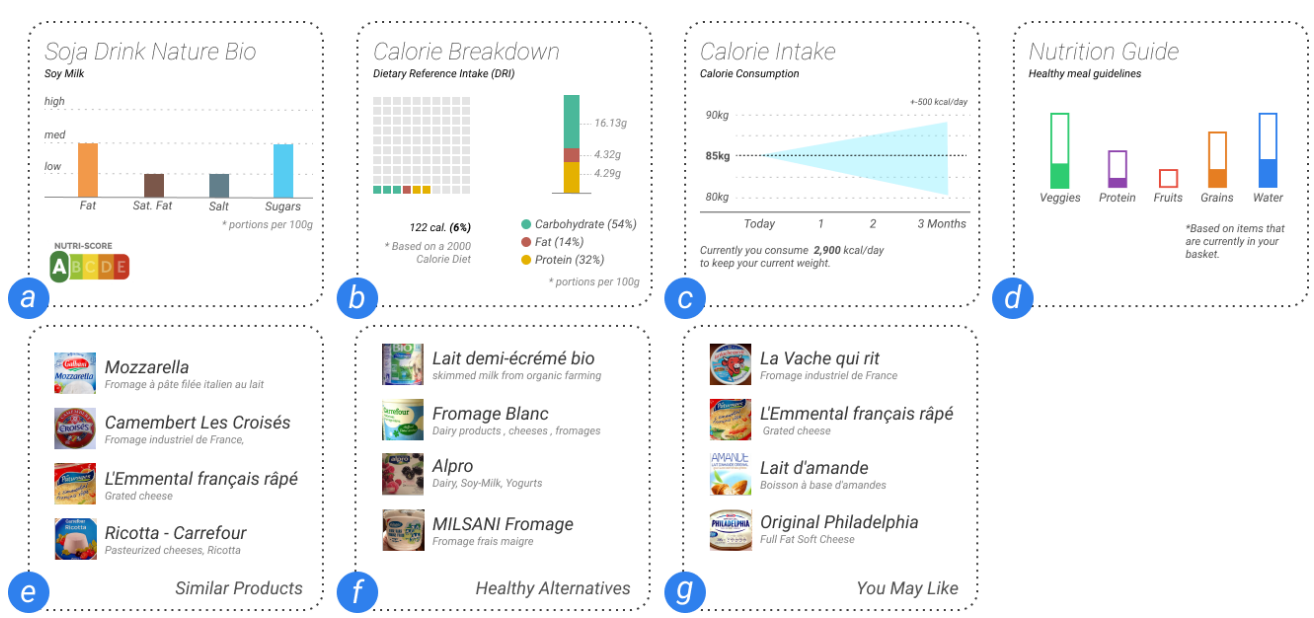

Figure 2. Visual components used in PHARA. a) Nutrition Levels, b) Calorie Breakdown, c) Calorie Intake, d) Nutrition Guide, e) Similar Products, f) Healthy Alternatives and g) You May Like.

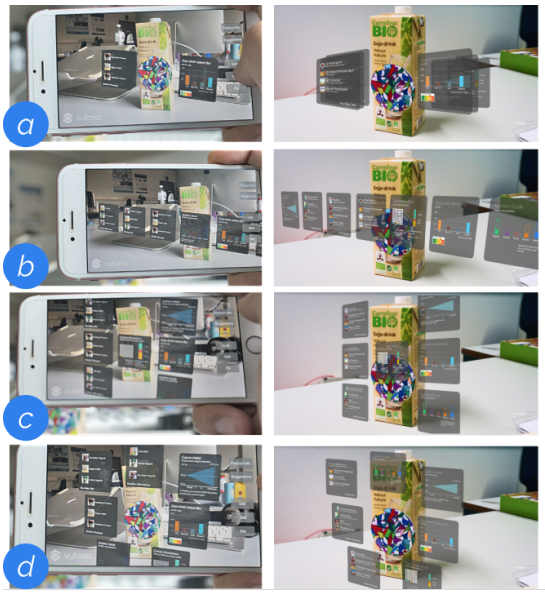

Figure 3. Different PHARA layouts. Left: Handheld. Right: HMD. a) stack, b) list c) grid and d) pie

balanced meal. When participants add products to their basket, we update the bar chart to indicated if they have enough of each of the categories.

Figure 2e, Similar Products Card: this card shows the top-four recommendations of similar products based on a product and its descriptions.

Figure 2f, Healthy Alternatives Card: using information from the food products database, we filtered out unhealthy products based on their nutri-score. Using the similarity index, we rank the top-four most similar products based on their description and ingredients.

Figure 2g, You May Like Card: using information from the food products database and the profile of the user, we recommended similar products based on those related to their interest and the current product they are holding in their hand.
Next, we designed and implemented four different layouts, as presented in Figure 3. In the next paragraphs, we describe all the layouts implemented in the system.

Figure 3a, Stack Layout: the stack layout uses an affordance of AR, namely the ability to use the three-dimensional space to organize information. This layout organizes the content by groups on different layers of information next to the object. Users can explore the content by swapping the position of the cards, bringing them to the front when they are required. Cards are represented with a light opacity, allowing to see through the layers.

Figure 3b, List Layout: the list layout positions all the cards horizontally on one row in front of the product. We designed the list to take advantage of the horizontal space of the field of view of the HMD and the width screen size of handheld devices. Users can scan through all of the cards by moving the target, from left to right and vice versa.

Figure 3c, Grid Layout: the grid layout has been applied to many user interfaces to organize and display content. Rows and columns organize all the cards arranged next to each other. The typical presentation is to align the items horizontally one by one. The layout presents a $2 \times 3$ grid, representing two columns and three rows.

Figure 3d, Pie Layout: another typical two-dimensional layout design is to place the items in a compass format. In the pie layout, items are placed as a circle around the target at equal distance from the center. The pie layout could offer a novel alternative and potentially more efficient design to be studied, since it is attributed to the capability of short target-seeking time and low error in selection [27]. The reason is that it would support users to have a quicker overview of 


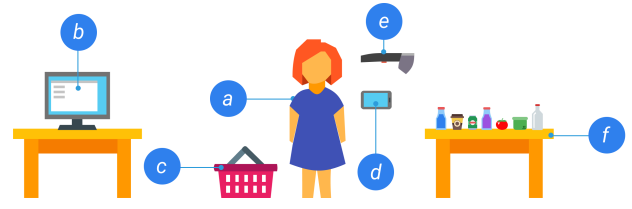

Figure 4. Scenario: a) Participant b) PC for creating a profile c) Basket to keep products during tasks d) Handheld device e) HMD device f) Food products.

all displayed items, as the interface consumes greater width but less height. Also, it allows users to see the items faster as the distance between elements is reduced. We implemented two different interaction systems for the applications. Based on feedback from previous studies, we decided to implement PHARA's HMD interaction using a Nintendo Joy-Con controller, instead of voice commands or hand gestures. The reason behind this is that in our early prototypes, participants reported feeling uncomfortable with the interaction when trying to switch between different layouts, and discomfort when giving voice commands in public spaces. Also, we considered the use of the finger gesture, but the number of interactions required and the close positioning of the food product to the person made the interaction uncomfortable to use for early users of PHARA. Overall, the controller made the interaction more comfortable for users in the preliminary studies. On the other hand, for the PHARA handheld experience, we included different on-screen buttons to let users perform the same actions with the different layouts.

\section{Study Design}

To answer our research question, we designed a within-subjects study to investigate the effects of layouts on decision-making of AR experiences. The overview of our scenario is presented in Figure 4. First, we collected a total of 50 food products including sugary drinks, water, vegetables, bread, meat and snacks to be used in the study. Then, we created two tasks based on real life scenarios. We explain the details in the following paragraphs. The tasks were designed in a way that required users to use all of the designed cards in the system's interface. Users had to complete each task using either the HMD or the smartphone. The order in which the two devices were presented was rotated using a counterbalancing measure meanwhile that of four different card layouts were rotated randomly. Therefore, half of the participants completed task one with the HMD followed by task two with the smartphone, whereas the other half completed task one with the smartphone followed by task two with the HMD. At the beginning of the study, participants had to create a profile, using a computer placed in the lab, indicating the necessary personal information to use the system. Next, the system was explained to them with a brief introduction and tutorial on how to use the HoloLens, the controllers, the mobile app, the layouts and each of the cards. An HMD or a smartphone was then provided to them depending on the counterbalanced order. A basket was also provided to the participants where they had to collect food products when completing the tasks. They were asked to think aloud their decisions and experience. Once completed, the participants continued with the second device and task. After using each device, a questionnaire was provided to capture the participants' perceived experience with the device. A total of 28 participants $(1 \mathrm{~F}, 27 \mathrm{M})$ were recruited via word-of-mouth. Ages ranged from 22 to $38(\mathrm{M}=25.81$, $\mathrm{SD}=4.57$ ). Participants were rewarded with chocolates and sweets in appreciation of their time.The two tasks that were used in this study are as follows:

Task 1: there were four major steps that each participant had to complete in task one. These were as follows:

1. Select two products that you would like to have for dinner, and put them in your basket.

2. Select two similar products to the ones you selected before.

3. Select and replace two alternatives to the products you have previously selected.

4. Reflect on the products that you have in your basket. Considering the number of calories, the number of products, and information on each product, do you think this would be a healthy meal? Replace products when you see a need to do so.

Task 2: similar to task one, participants had to complete the four steps again. Except for the first step, all the remaining steps were exactly the same:

1. Imagine that you have some friends coming over. Using the AR application, select two products that you would like to have for dinner together with your friends, and put them in the basket.

To answer our research question, we logged data from the AR interfaces usage. We calculated objective measures such as the time spent and the number of actions while using each layout as a measure of objective effort. Following our research question, we also surveyed the participants about their subjective perceptions, based on technology acceptance model (TAM). The TAM questionnaire has been used to assess the acceptance of AR applications [28]. However, we are also interested in measuring the perceived workload 
from the participants when using each of the different layouts. The NASA task load index (NASA-TLX) [29] is tool previously used in AR applications to assess the perceived workload during task applications. For all of the questionnaire items, users had to indicate on seven-point Likert scales to what extent they agreed.

\section{Results}

The average completion time of the study for each participant was about 32 minutes. First, we analyze the interaction of the participants with the devices using the objective measures. Then, we investigate the results of the subjective data from the answers of the participants in the posterior questionnaires. We report the layout results separately for each device, the PHARA HMD, and PHARA handheld, as well as how they compare to each other. Lastly, we present the user comments derived from the think-aloud protocol.

Objective measures: to investigate further the results of our study, a repeated measures ANOVA was conducted with a Bonferroni post-hoc analysis as a follow-up. The traces of information revealed that participants tended to select more healthy food products when asked to reflect on their basket products during the fourth task, compared to the number of healthy products they started within the first task. The analysis also revealed that the stack layout required a significantly higher number of actions and time spent to complete the tasks compared to the list and the pie layouts $(p<.05)$, in both the PHARA handheld and the HMD experience.

Subjective measures: we inquired about the subjective perceptions of the participants with the layouts and AR experiences, particularly towards the acceptance of AR as a new technology and the impact on cognitive load for decision-making. In this section, we analyze the results from TAM and raw NASA-TLX questionnaires, breaking down each of the subjective measures. We report the results separated for the two AR experiences. A non-parametric Friedman's ANOVA test procedure was conducted to investigate the differences between the subjective measures. Results of the subjective measures are presented in Figure 5.

PHARA handheld layouts: we summarize the results from the smartphone device in Table 1 . We found that participants had a better experience when using the grid and the pie layouts. Participants indicated that the grid and pie layouts were significantly more adequate and easy to use during their experiences. They significantly enjoyed these layouts more, felt more confident, and intended to use these layouts. In addition, participants indicated that the stack and the list layouts were more physically and mentally demanding, leading them to

\begin{tabular}{|c|c|c|c|c|c|}
\hline \multicolumn{2}{|c|}{ PHARA Handheld } & Grid & Stack & List & Pie \\
\hline \multirow{5}{*}{ 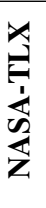 } & Physical Demand & $3 * * *$ & 4 & 5 & $2 * * *$ \\
\hline & Mental Demand & $3 *$ & 4.5 & 3.5 & $3 * *$ \\
\hline & Overall Perf. & 3.5 & 3 & 4 & 2.5 \\
\hline & Effort & $3 * * *$ & 4 & 4 & $3 * * *$ \\
\hline & Frustration & $2.5 * * *$ & 3 & 4 & $2 *$ \\
\hline \multirow{5}{*}{$\sum_{\S}$} & Confidence & $5 * *$ & 3 & 4 & $5 * *$ \\
\hline & Interface Adeq. & $5 * *$ & 3 & 4 & $6 * *$ \\
\hline & Ease of Use & $5 * *$ & 3 & 3 & $5 * * *$ \\
\hline & Enjoyment & $5 * *$ & 3 & 3 & $5.5 * * *$ \\
\hline & Use Intention & $5.5 * * *$ & 2.5 & 3 & $6 * * *$ \\
\hline \multicolumn{2}{|c|}{ PHARA HMD } & Grid & Stack & List & Pie \\
\hline \multirow{5}{*}{ 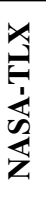 } & Physical Demand & 4 & 3 & 4 & 3.5 \\
\hline & Mental Demand & 4 & 3 & $4 * * *$ & 3 \\
\hline & Overall Perf. & 4 & 3 & 4 & 4 \\
\hline & Effort & 3.5 & 3.5 & 4 & 5 \\
\hline & Frustration & 3.5 & 3 & 3.5 & 3 \\
\hline \multirow{5}{*}{$\sum_{\xi}$} & Confidence & 4 & 5 & 4 & 5 \\
\hline & Interface Adeq. & 4 & 5 & 4 & 5 \\
\hline & Ease of Use & 4 & 5 & 4 & 5 \\
\hline & Enjoyment & 4 & $5 * *$ & 4 & 4.5 \\
\hline & Use Intention & 4 & 5 & 3.5 & 5 \\
\hline
\end{tabular}

Table 1. Results from handheld and HMD studies: median values from the qualitative feedback. ${ }^{*} p<0.05 ; * * p<0.01 ; * * * p<0.001$

a significantly greater level of frustration. They also indicated that the effort they had to put in the stack and list layouts was significantly greater compared to the grid and the pie layouts.

PHARA HMD Layouts: we summarize the results from the PHARA HMD experience in Table 1. Participants tended to rank the stack and pie layouts significantly higher with respect to enjoyment and intention to use factors. Participants also indicated that the list layout was significantly more demanding than the stack layout.

PHARA handheld vs PHARA HMD: a Wilcoxon signed-rank test was used to compare the results of technology acceptance towards the two PHARA experiences. Results are presented in Figure 6. Our analysis indicates that participants found the PHARA handheld experience significantly more enjoyable compared to the HMD experience $(p<.05)$. Moreover, the perceived usefulness and perceived ease of use was higher for the handheld experience $(r=0.5, p<$ .05) than for the HMD experience. The stack layout showed to be significantly more physically and mentally demanding with the PHARA handheld experience $(p<$ .05). Participants felt more confident with the pie and the grid layouts. Results also indicate that these layouts were significantly more adequate and easy 


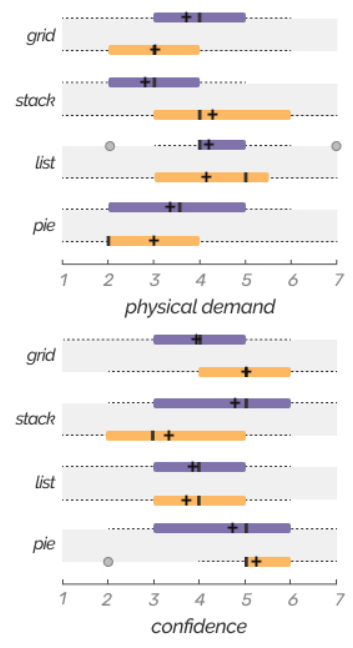

- PHARAHMD PHARA Handheld

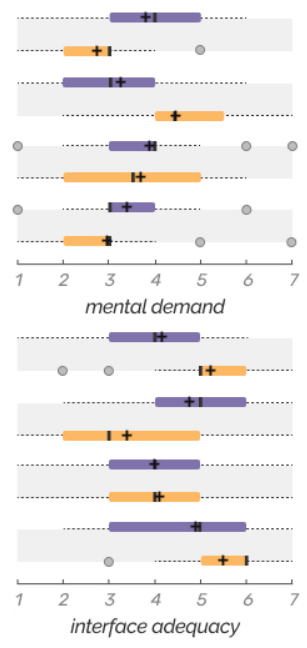

ld

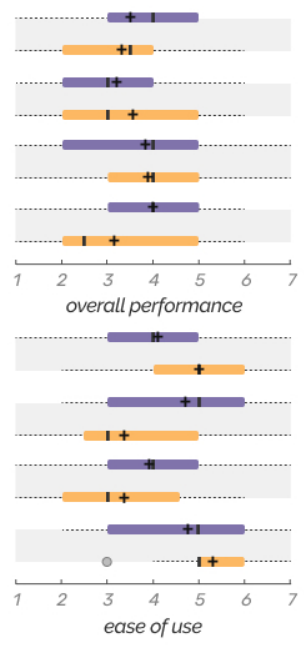

ease of use

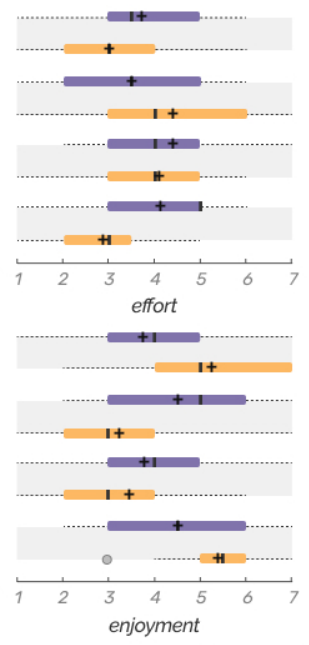

(1 = strongly disagree, 7 = strongly agree)

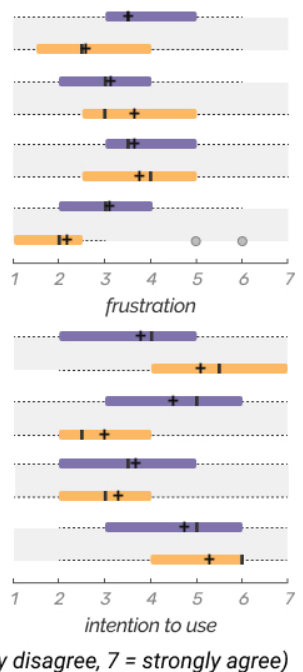

Figure 5. Thoughts about the layouts used by device. Top: perceived workload, Bottom: technology acceptance.

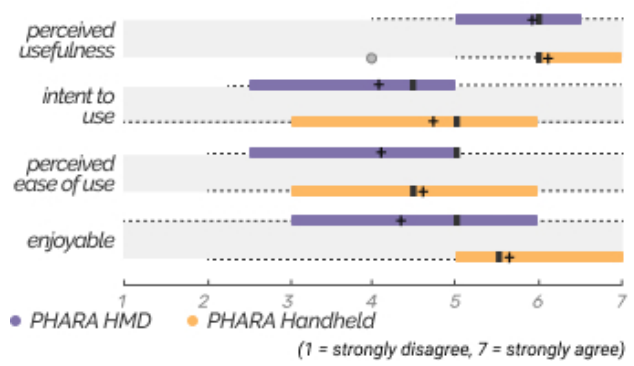

Figure 6. Thoughts of the participants towards technology acceptance.

to use $(p<.05)$, providing a significantly greater enjoyment and use intention in the handheld experience $(p<.05)$. Comparing the PHARA HMD and handheld experiences, participants thought that the grid and the pie layouts were significantly more physically and mentally demanding $(p<.05)$ in the HMD experience. Even though the pie showed a significantly better overall performance $(p<0.5)$, participants indicated it required a significantly higher effort, together with the grid $(p<.05)$, leading them to feel significantly more $(p<.05)$ frustrated when using these layouts in the HMD experience. Moreover, during the HMD experience, participants felt that the stack layout was significantly more adequate, enjoyable and easy to use $(p<.05)$, significantly increasing their intentions to use it $(p<.05)$, compared to the handheld experience.

Comments from the participants: during the PHARA HMD experience, most of the comments were related to the hardware limitations of the device. Several participants indicated the field of view as a potential limitation: "I would keep the layout as narrow as possible because of the field of view of the HoloLens." Some participants suggested to adjust the interface and keep it as minimal as possible to improve the usability. A participant referred to the image tracking and the physical demand required by the device: "Arm length is a problem... (the food product) ...needs to be closer. view-port is very small, had pain in the back after a while by holding the item so far." Given the hardware limitations of the HoloLens, participants pointed out the potential of the stacked layout. "Field of view of HoloLens is responsible for the bad scores. Only with the stacked view everything was visible." Some participants preferred the pie layout because of the positioning of the card components around the target. "I think pie layout makes the best use of the available space." However, for some participants the pie layout was also limited by the hardware: "with pie layout, the top ones can be hard to see.” Apparently, the list was the less popular layout among the participants. However, a participant indicated its usefulness when showing all the information at the same time. "For me, the list was best because you could see most info boards at the same time (stack was also okay but took a bit more time)." Some participants indicated their preference when comparing the grid against the pie layout. "Grid was a bit distracting because you could see some boards only partially." Some participants also commented towards the expensive use of the stack layout. "A button to go back to the previous screen in the stack rotation, instead of having to go through all the screens to get back to the previous one." On the other hand, during the PHARA handheld experience, participants indicated some usability issues while holding the device with the hand. "The stack layout was more difficult to use with 
a single hand; therefore, I preferred the other options better." Some participants thought that the tracking was very limited in the list. "The list layout has the problem that you want to go close to the text, but then it loses track of the tracker allows easier comparison by tagging several objects as favorites.” A participant suggested a lock feature to enable zoom-in options: "Maybe have a way of fixing, locking on a product, so you can zoom in on charts in the non-stacked layouts without switching to another product." Some participants pointed that the list was long, and that other layouts made better use of the screen space. "The List was sometimes quite long. Therefore, the grid and pie are better since these layouts show the information in a compact way".

\section{Discussion}

We compare the results in order to answer our research question: Regarding subjective and objective factors: what are the benefits and tradeoffs of using popular layouts such as, stack, grid, list and pie in AR applications within Handheld and HMD to support decision-making?

We found that the stack layout may be useful to organize virtual content as layers in the physical space. The stack layout was the most useful for interacting with the reduced field of view of the HoloLens at the cost of more actions and time spent, leading to a higher physical demand. However, the stack layout did not feel adequate during the handheld experience. Participants pointed out that the stack was a practical layout to organize the information, but they felt more confident with the other layouts where they could see the information all at once. The grid layout tended to have a more positive score in the handheld experience. It was the second most used layout by the participants where they spent most of their time and actions when solving the tasks. Participants indicated that the grid was an excellent choice for the handheld device, requiring less physical and mental demand compared to the stack.

When looking at the pie layout, participants often compared it against the grid layout because of their similarities. However, when solving the tasks, participants noted that the pie had a better use of the vertical space. Both the grid and the pie tended to score high in terms of confidence in the decisions of the participants, also indicating that these were adequate, enjoyable and easy to use layouts for the handheld device. The pie also tended to show lower scores towards required effort and frustration.

The list layout was the least preferred layout among the participants, because of its large size. Some participants appreciated the list as a layout to see everything at once. However, it was not as useful for exploratory zoom-in actions. Although interesting results have been obtained, there are a few limitations that should be articulated. Despite the fact that we performed a review of suitable layouts for designing our application, and selected the most popular ones, other layouts might be also attractive to analyze. The HoloLens might have a substantial impact on the final results, since the narrow field of view had an strong effect on the preferred layout selection. Further work will extend the exploration to different devices. Moreover, the sample size is a limitation of our study, as well as the limited number of female participants. We intend to extend the number of participants in further studies.

\section{Conclusions}

We have described the design and implementation of PHARA, an AR system that shows different information cards around physical food products in the real world. PHARA allows users to make informed decisions, and resulted in selecting healthier food products. We explored and evaluated the utility of four different layouts to present information and assist users in a decision-making scenario. Our findings in this study include implications on the design of AR applications for handheld and HMD experiences. We found that the stack visualization performs better with HMD devices with a limited field of view, like the HoloLens, at the cost of some usability affordances. We also discovered that our results are comparable to those of [9], even though they compared different layouts in a desktop application for recommender systems. In our study, we found that the grid and pie layouts perform better than the other layouts in the handheld devices, allowing participants to explore more products with more confidence, enjoyability and less effort. Ultimately, one of the major goals is to be able to influence grocery store shopping and in-restaurant purchase behavior. Future work will evaluate the potential of our approach in such real-life settings.

\section{Acknowledgments}

Part of this work has been supported by the KU Leuven Research Council (grant agreement C24/16/017) and the Research Foundation Flanders (FWO, grant agreement no. G0C9515N).

\section{References}

[1] R. T. Azuma, "A survey of augmented reality," Presence: Teleoperators and virtual environments, vol. 6, no. 4, 
pp. 355-385, 1997.

[2] D. Van Krevelen and R. Poelman, "A survey of augmented reality technologies, applications and limitations," International Journal of Virtual Reality, vol. 9, no. 2, p. 1, 2010.

[3] J. Grubert, M. Pahud, R. Grasset, D. Schmalstieg, and H. Seichter, "The utility of magic lens interfaces on handheld devices for touristic map navigation," Pervasive and Mobile Computing, vol. 18, pp. 88-103, 2015.

[4] M. Serino, K. Cordrey, L. McLaughlin, and R. L. Milanaik, "Pokémon go and augmented virtual reality games: a cautionary commentary for parents and pediatricians," Current opinion in pediatrics, vol. 28, no. 5, pp. 673-677, 2016.

[5] D. Brohm, N. Domurath, V. Glanz-Chanos, and K. G. Grunert, "Future trends of augmented reality," in Augmented reality for food marketers and consumers, pp. 1681-1685, Wageningen Academic Publishers, 2017.

[6] M. Cecchini and L. Warin, "Impact of food labelling systems on food choices and eating behaviours: a systematic review and meta-analysis of randomized studies," Obesity reviews, vol. 17, no. 3, pp. 201-210, 2016.

[7] V. Heun, S. Kasahara, and P. Maes, "Smarter objects: using ar technology to program physical objects and their interactions," in CHI'13 Extended Abstracts on Human Factors in Computing Systems, pp. 961-966, ACM, 2013.

[8] J. B. Madsen, M. Tatzqern, C. B. Madsen, D. Schmalstieg, and D. Kalkofen, "Temporal Coherence Strategies for Augmented Reality Labeling," IEEE Transactions on Visualization and Computer Graphics, vol. 22, no. 4, pp. 1415-1423, 2016.

[9] L. Chen and H. K. Tsoi, "Users' decision behavior in recommender interfaces: Impact of layout design," in RecSys' 11 Workshop on Human Decision Making in Recommender Systems, 2011.

[10] A. C. Valdez, M. Ziefle, K. Verbert, A. Felfernig, and A. Holzinger, "Recommender systems for health informatics: State-of-the-art and future perspectives," in Machine Learning for Health Informatics, pp. 391-414, Springer, 2016.

[11] M. Wiesner and D. Pfeifer, "Health recommender systems: concepts, requirements, technical basics and challenges," International journal of environmental research and public health, vol. 11, no. 3, pp. 2580-2607, 2014.

[12] R. G. Farrell, C. M. Danis, S. Ramakrishnan, and W. A. Kellogg, "Intrapersonal retrospective recommendation: lifestyle change recommendations using stable patterns of personal behavior," in Proc. of the First International Workshop on Recommendation Technologies for Lifestyle Change (LIFESTYLE 2012), Dublin, Ireland, p. 24, 2012.

[13] H. Roitman, Y. Messika, Y. Tsimerman, and Y. Maman, "Increasing patient safety using explanation-driven personalized content recommendation," in Proc. of the 1st ACM International Health Informatics Symposium, pp. 430-434, ACM, 2010.

[14] D. Elsweiler, M. Harvey, B. Ludwig, and A. Said, "Bringing the "healthy" into food recommenders," in 2nd International Workshop on Decision Making and Recommender Systems, pp. 33-36, 2015.
[15] M. Rokicki, E. Herder, and E. Demidova, "What's on my plate: Towards recommending recipe variations for diabetes patients.," in UMAP Workshops, 2015.

[16] Y. van Pinxteren, G. Geleijnse, and P. Kamsteeg, "Deriving a recipe similarity measure for recommending healthful meals," in Proc. of the 16th international Conf. on Intelligent user interfaces, pp. 105-114, ACM, 2011.

[17] P. Achananuparp and I. Weber, "Extracting food substitutes from food diary via distributional similarity," in Proc. of the 2016 Workshop on Engendering Health with RecSys, RecSys '16, pp. 1-4, ACM, 2016.

[18] S. Shekar, P. Nair, and A. S. Helal, "igrocer: a ubiquitous and pervasive smart grocery shopping system," in Proc. of the 2003 ACM symposium on Applied computing, pp. 645-652, ACM, 2003.

[19] J. Ahn, J. Williamson, M. Gartrell, R. Han, Q. Lv, and S. Mishra, "Supporting healthy grocery shopping via mobile augmented reality," ACM Transactions on Multimedia Computing, Communications, and Applications (TOMM), vol. 12, no. 1s, p. 16, 2015.

[20] C. Siawsolit, S. Seepun, J. Choi, A. Do, and Y. Kao, "Personalized assistant for health-conscious grocery shoppers," in International Conf. on Persuasive Technology, pp. 95-106, Springer, 2017.

[21] M. Teyssier, G. Cliquet, and S. Richir, "ArLive: Unified Approach of Interaction Between Users, Operable Space and Smart Objects," Proc. of the 2016 Virtual Reality International Conf., pp. 21:1-21:5, 2016.

[22] F. Gutiérrez, B. Cardoso, and K. Verbert, "Phara: a personal health augmented reality assistant to support decision-making at grocery stores," in Second International Workshop on Health Recommender Systems, p. 10, 2017.

[23] M. El-Dosuky, M. Rashad, T. Hamza, and A. El-Bassiouny, "Food recommendation using ontology and heuristics," in International Conf. on Advanced Machine Learning Technologies and Applications, pp. 423-429, Springer, 2012.

[24] M. D. Mifflin, S. T. St Jeor, L. A. Hill, B. J. Scott, S. A. Daugherty, and Y. O. Koh, "A new predictive equation for resting energy expenditure in healthy individuals.," The American journal of clinical nutrition, vol. 51, no. 2, pp. 241-247, 1990.

[25] P. Ducrot, C. Julia, C. Méjean, E. Kesse-Guyot, M. Touvier, L. K. Fezeu, S. Hercberg, and S. Péneau, "Impact of different front-of-pack nutrition labels on consumer purchasing intentions: a randomized controlled trial," American journal of preventive medicine, vol. 50, no. 5, pp. 627-636, 2016.

[26] W. Willett, Eat, drink, and be healthy: the Harvard Medical School guide to healthy eating. Free Press, 1 ed., July 2011.

[27] S. Lin, H. F. Cheng, W. Li, Z. Huang, P. Hui, and C. Peylo, "Ubii: Physical world interaction through augmented reality," IEEE Transactions on Mobile Computing, vol. 16, no. 3, pp. 872-885, 2017.

[28] T. Olsson, T. Kärkkäinen, E. Lagerstam, and L. Ventä-Olkkonen, "User evaluation of mobile augmented reality scenarios," Journal of Ambient Intelligence and Smart Environments, vol. 4, no. 1, pp. 29-47, 2012.

[29] S. G. Hart and L. E. Staveland, "Development of nasa-tlx (task load index): Results of empirical and theoretical research," Advances in psychology, vol. 52, pp. 139-183, 1988. 